Discussion Paper No. 699

\title{
SECURE IMPLEMENTATION IN ECONOMIES WITH INDIVISIBLE OBJECTS AND MONEY
}

\author{
Yuji Fujinaka \\ Takuma Wakayama
}

October 2007

The Institute of Social and Economic Research Osaka University

6-1 Mihogaoka, Ibaraki, Osaka 567-0047, Japan 


\title{
Secure Implementation in Economies with Indivisible Objects and Money*
}

\author{
Yuji Fujinaka $^{\dagger \ddagger} \quad$ Takuma Wakayama ${ }^{\S}$
}

October 5, 2007

\begin{abstract}
This paper studies the application of the notion of secure implementation (Cason, Saijo, Sjöström, and Yamato, 2006; Saijo, Sjöström, and Yamato, $2007)$ to the problem of allocating indivisible objects with monetary transfers. We propose a new domain-richness condition, termed as minimal richness. We then establish that on any minimally rich domain, only constant social choice functions are securely implementable.
\end{abstract}

Keywords: Secure implementation, Dominant strategy implementation, Nash implementation, Indivisible goods, Strategy-proofness.

JEL codes: C72, C78, D61, D63, D71.

${ }^{*}$ We would like to thank an anonymous referee and the editor, Eric Maskin for helpful comments and suggestions. We are also grateful to Jun Iritani, Kaoru Ishiguro, Noritsugu Nakanishi, Tatsuyoshi Saijo, Toyotaka Sakai, and Shohei Takagi for their helpful comments and discussions.

${ }^{\dagger}$ JSPS Research Fellow.

${ }^{\ddagger}$ Correspondence: ISER, Osaka University, 6-1 Mihogaoka Ibaraki Osaka 567-0047, JAPAN; +81-6-6879-9179; fujinaka@iser.osaka-u.ac.jp; http://www.geocities.jp/yuji_fujinaka/

${ }^{\S}$ Faculty of Urban Liberal Arts, Tokyo Metropolitan University, 1-1 Minami-Osawa, Hachioji, Tokyo 192-0397, JAPAN; t_waka@center.tmu .ac.jp; http://www.geocities.jp/takuma_wakayama/ 


\section{Introduction}

In this paper, we study the problem of allocating indivisible objects when monetary transfers are possible. ${ }^{1}$ We assume that each agent consumes one and only one indivisible object. To illustrate the problem, let us consider a situation where a group of regions jointly construct various types of public facilities. When a region accepts a desirable facility such as a library, it can enjoy a utility. On the other hand, when another region accepts an undesirable facility such as a garbage disposal facility, it must bear a disutility. Thus, there arises a need to assign such facilities among regions and simultaneously distribute money to compensate for the differences in the characteristics of facilities. This problem also applies to the allocation of houses or rooms or to the assignment of jobs. In this model, each allocation consists of an assignment of indivisible objects and the specification of an amount of money for each agent. A social choice function (or direct revelation mechanism) is the function that associates an allocation with each preference profile.

Strategy-proofness, which states that no one gains by preference misrepresentation, is a primary requirement in mechanism design. Saijo, Sjöström, and Yamato (2007) indicate that many strategy-proof mechanisms (to be exact, the direct revelation mechanisms associated with strategy-proof social choice functions) admit multiple Nash equilibrium outcomes that are different from the "true" outcome, thereby making these mechanisms somewhat ineffective. ${ }^{2}$ Therefore, Cason, Saijo, Sjöström, and Yamato (2006) and Saijo, Sjöström, and Yamato (2007) develop a new concept, namely, secure implementation. A social choice function is securely implementable if there exists a mechanism that implements it through dominant strategy equilibria and if the set of dominant strategy equilibrium outcomes coincides with the set of Nash equilibrium outcomes. ${ }^{3}$

Our purpose is to examine the social choice functions that are securely implementable in our economies. ${ }^{4}$ We provide a new mild domain-richness condition, termed as minimal richness. Then, we show that on any minimally rich domain, only constant social choice functions are securely implementable.

This paper is organized as follows: Section 2 defines the basic notions as well as the concept of secure implementation, and introduces the concept of minimal richness. Section 3 provides the primary result. Section 4 concludes the paper.

\footnotetext{
${ }^{1}$ Basic models of economies with indivisible objects and monetary transfers were provided by Svensson (1983) and Alkan, Demange, and Gale (1991).

${ }^{2}$ The results of the experiments conducted by Cason, Saijo, Sjöström, and Yamato (2006) support this idea.

${ }^{3}$ In other words, secure implementation signifies double implementation in dominant strategy equilibria and Nash equilibria. Fujinaka and Sakai (2006) present a study of Nash implementation in our economies.

${ }^{4}$ In several economic environments, some results on secure implementation have been established. For example, Saijo, Sjöström, and Yamato (2007) for economies with a public good and money, and Bochet and Sakai (2007) for allotment economies with single-peaked preferences.
} 


\section{Model}

\subsection{Basic Notion}

Let $I \equiv\{1,2, \ldots, n\}$ be a finite set of agents. There are finite types of indivisible objects $T \equiv\{1,2, \ldots, s\}$ and a divisible good which is called "money." For each type $t \in T$, let $\alpha_{t} \geq 1$ be the integer number of type- $t$ indivisible objects. In this paper, we assume the following.

A1. $\sum_{t \in T} \alpha_{t} \geq n$.

A2. Each agent $i \in I$ has the consumption space denoted by $T \times \mathbb{R}$.

A1 states that the total number of indivisible objects is at least as great as the number of agents, while A2 states that each agent consumes precisely one indivisible object and an amount of money. A consumption bundle in $T \times \mathbb{R}$ is denoted by $\left(t, m_{i}\right)$, where $t \in T$ denotes a type- $t$ object, and $m_{i} \geq 0\left(m_{i}<0\right)$ denotes the amount of money that agent $i$ receives (pays).

It should be noted that A1 allows us to deal with the case where the number of indivisible objects are less than the number of agents. To observe this, let us consider a situation where there is the only one type of indivisible object and the number of objects of this type is one, i.e., $T=\{1\}$ and $\alpha_{1}=1$. In this situation, all the agents, except one, cannot receive any indivisible object. Therefore, they cannot enjoy any utility from the assignment of the indivisible object. Now, we additionally consider a fictitious object, say, a "null" object, from whose type no agent can gain any utility. If we regard the null object as a type- 2 object, we can rewrite the situation as follows: $T^{\prime}=\{1,2\}, \alpha_{1}=1$, and $\alpha_{2}=n-1$. If we interpret that the agents who are initially not assigned a type- 1 object receive a type-2 object, each analysis on the new situation corresponds to one on the initial situation. Therefore, based on our assumptions, we can deal with the situation where there is only one indivisible object.

Each agent's preferences over $T \times \mathbb{R}$ are quasi-linear with respect to money. If agent $i$ consumes a type- $t$ object and her monetary transfer is $m_{i} \in \mathbb{R}$, then her utility level is $v_{i}(t)+m_{i}$, where $v_{i}(t) \in \mathbb{R}$ is agent $i$ 's valuation for a type- $t$ object. Let $V_{i}(t) \subseteq \mathbb{R}$ be a non-empty set of $i$ 's valuation for a type- $t$ object. Agent $i$ 's valuation vector is $v_{i} \equiv\left(v_{i}(t)\right)_{t \in T} \in \mathbb{R}^{T}$. Let $V_{i} \equiv \prod_{t \in T} V_{i}(t) \subseteq \mathbb{R}^{T}$ be the set of valuation vectors for agent $i$. We denote a profile of valuation vectors by $v \equiv\left(v_{1}, v_{2}, \ldots, v_{n}\right)$, and the set of profiles of valuation vectors by $V \equiv \prod_{i \in I} V_{i}$. The set $V$ is called a domain. Since agents' preferences are usually unknown to a mechanism designer, the following assumption is natural and reasonable.

A3. There exist $i \in I$ and $t \in T$ such that $\left|V_{i}(t)\right| \geq 2 .^{5}$

We often denote $I \backslash\{i\}$ by " $-i$," and $I \backslash\{i, j\}$ by " $-i, j$." With this notation, $\left(v_{i}^{\prime}, v_{-i}\right)$ is the profile of valuation vectors where agent $i$ has $v_{i}^{\prime}$ and agent $j \neq i$ has $v_{j}$. We define $\left(v_{i}^{\prime}, v_{j}^{\prime}, v_{-i, j}\right)$ similarly.

\footnotetext{
${ }^{5}$ Given a set $A$, we denote the cardinality of $A$ by $|A|$.
} 
An assignment is a list $\sigma \equiv\left(\sigma_{1}, \sigma_{2}, \ldots, \sigma_{n}\right)$ such that

$$
\begin{aligned}
& \sigma_{i} \in T \text { for each } i \in I, \\
& \left|\left\{i \in I: \sigma_{i}=t\right\}\right| \leq \alpha_{t} \text { for each } t \in T .
\end{aligned}
$$

Given $i \in I, \sigma_{i}$ is the object assigned to agent $i$, and $\sigma_{i}=t$ means that agent $i$ receives a type- $t$ object. A monetary transfer is a list $m \equiv\left(m_{1}, m_{2}, \ldots, m_{n}\right)$ such that $m_{i} \in \mathbb{R}$ for each $i \in I$. Note that we do not require any feasibility constraint on monetary transfers. If we establish an impossibility result, this strengthens the result. An allocation is a list $(\sigma, m) \in T^{I} \times \mathbb{R}^{I}$ such that $\sigma$ is an assignment and $m$ is a monetary transfer.

A social choice function on a domain $V$ is a function $f: V \rightarrow T^{I} \times \mathbb{R}^{I}$ associating with each profile of valuation vectors $v \in V$ an allocation $f(v) \equiv\left(f_{i}(v)\right)_{i \in I} \in T^{I} \times \mathbb{R}^{I}$. For convenience, given $v \in V$ and $i \in I$, we write $f_{i}(v) \equiv\left(\sigma_{i}(v), m_{i}(v)\right)$.

\subsection{Secure Implementation}

A social choice function is securely implementable if there exists a mechanism that simultaneously implements the social choice function in dominant strategy equilibria and Nash equilibria. ${ }^{6}$ In this paper, we would like to characterize the class of securely implementable social choice functions in our economies. Saijo, Sjöström, and Yamato (2007) provide a characterization of the class in the general model ${ }^{7}$ : A social choice function is securely implementable if and only if it satisfies strategyproofness and the rectangular property.

Strategy-proofness: For each $v \in V$, each $i \in I$, and each $v_{i}^{\prime} \in V_{i}, v_{i}\left(\sigma_{i}(v)\right)+$ $m_{i}(v) \geq v_{i}\left(\sigma_{i}\left(v_{i}^{\prime}, v_{-i}\right)\right)+m_{i}\left(v_{i}^{\prime}, v_{-i}\right)$.

Rectangular Property: For each $v, v^{\prime} \in V$, if $v_{i}\left(\sigma_{i}\left(v^{\prime}\right)\right)+m_{i}\left(v^{\prime}\right)=v_{i}\left(\sigma_{i}\left(v_{i}, v_{-i}^{\prime}\right)\right)+$ $m_{i}\left(v_{i}, v_{-i}^{\prime}\right)$ for each $i \in I$, then $f\left(v^{\prime}\right)=f(v)$.

\subsection{Minimal Richness}

We now present a new domain-richness condition, called minimal richness. In order to formally define minimal richness, we use the following notation. Given $i \in I$, $v_{i} \in V_{i}$, and $t^{\prime}, t^{\prime \prime} \in T$, let $\Delta v_{i}\left(t^{\prime} ; t^{\prime \prime}\right) \equiv v_{i}\left(t^{\prime}\right)-v_{i}\left(t^{\prime \prime}\right)$.

Minimal Richness: For each $i \in I$, each $v_{i}^{\prime}, v_{i}^{\prime \prime} \in V_{i}$, each $t^{\prime}, t^{\prime \prime} \in T$, and each $M \in \mathbb{R}$, if $\Delta v_{i}^{\prime}\left(t^{\prime} ; t^{\prime \prime}\right)>M>\Delta v_{i}^{\prime \prime}\left(t^{\prime} ; t^{\prime \prime}\right)$, there exists $v_{i}^{*} \in V_{i}$ such that

(i) $\Delta v_{i}^{*}\left(t^{\prime} ; t^{\prime \prime}\right)=M$,

(ii) $\Delta v_{i}^{\prime}\left(t ; t^{\prime \prime}\right) \geq \Delta v_{i}^{*}\left(t ; t^{\prime \prime}\right)$ for each $t \in T \backslash\left\{t^{\prime}, t^{\prime \prime}\right\}$.

\footnotetext{
${ }^{6}$ See Saijo, Sjöström, and Yamato (2007) for the formal definition of secure implementation.

${ }^{7}$ Mizukami and Wakayama (2007) provide an alternative characterization of securely implementable social choice functions.
} 
Minimal richness requires that for any agent $i \in I$, any distinct valuation vectors $v_{i}^{\prime}, v_{i}^{\prime \prime} \in V_{i}$, any distinct types $t^{\prime}, t^{\prime \prime} \in T$, and any real number $M \in \mathbb{R}$, if the number $M$ lies in the range from $\Delta v_{i}^{\prime \prime}\left(t^{\prime} ; t^{\prime \prime}\right)$ to $\Delta v_{i}^{\prime}\left(t^{\prime} ; t^{\prime \prime}\right)$, there exists another valuation vector $v_{i}^{*} \in V_{i}$ satisfying the following: (i) $\Delta v_{i}^{*}\left(t^{\prime} ; t^{\prime \prime}\right)$ is equal to the number $M$, and (ii) the value of $t^{\prime \prime}$ for $v_{i}^{*}$, relative to any other types, is greater than or equal to that of $t^{\prime \prime}$ for $v_{i}^{\prime}$.

Since many reasonable domains satisfy minimal richness, it is considered as a mild requirement for domains in our model. The following domain, which is referred to as a box-shaped domain, is an example of a minimally rich domain. ${ }^{8}$ It roughly states that $V_{i}(t)$ takes the form of a certain interval for each $i \in I$ and each $t \in T$.

Box-shapedness: For each $i \in I$, each $t \in T$, and each $v_{i}(t), v_{i}^{\prime}(t) \in V_{i}(t)$ with $v_{i}^{\prime}(t) \geq v_{i}(t), v_{i}^{\prime \prime}(t) \in V_{i}(t)$ for each $v_{i}^{\prime \prime}(t) \in\left[v_{i}(t), v_{i}^{\prime}(t)\right]$.

Let $V^{U}, V^{G}$, and $V^{B}$ be domains such that any vector of valuations are possible, each type of indivisible object is "good" and each is "bad"; i.e., for each $i \in I$ and each $t \in T, V_{i}^{U}(t)=\mathbb{R}, V_{i}^{G}(t)=\mathbb{R}_{+}$, and $V_{i}^{B}(t)=\mathbb{R}_{-} .{ }^{9}$ By the definition of box-shapedness, these are box-shaped domains, and therefore, are minimally rich domains.

Given $t \in T$, if $V_{i}(t)=\{0\}$ for each $i \in I$, i.e., no agent gains any utility from the type- $t$ object, then type- $t$ objects can be interpreted as "null." Box-shaped domains include the case where some types are null objects, since the singleton is an interval. Thus, minimally rich domains also include the abovementioned case.

We will now provide an example of non-minimally rich domains.

Example 1. Let $I=\{1,2\}$ and $T=\{1,2\}$. Let $V^{*}$ be such that $V_{1}^{*}(2)=V_{2}^{*}(2)=$ $\{0\}, V_{1}^{*}(1)=\mathbb{R} \backslash\{1\}$, and $V_{2}^{*}(1)=\mathbb{R}$. To observe that $V^{*}$ is not minimally rich, let $v_{1}^{\prime}, v_{1}^{\prime \prime} \in V_{1}^{*}$ be such that $v_{1}^{\prime}(1)=2$ and $v_{1}^{\prime \prime}(1)=0$. Let $M=1$. Then, there is no $v_{1}^{*} \in V_{1}^{*}$ such that $v_{1}^{*}(1)-v_{1}^{*}(2)=M$.

The key is that $V_{1}^{*}$ is not connected. In the domain $V^{*}$, it is not permissible for agent 1 to have the valuation vector $(1,0)$; nevertheless, valuation vectors arbitrarily close to $(1,0)$ are permissible. In this sense, such non-connected domains are not reasonable. Therefore, we can consider that minimal richness is a mild requirement.

\section{Theorem}

In this section, we consider the set of securely implementable social choice functions whose domains are minimally rich. The next proposition characterizes the class of social choice functions satisfying strategy-proofness and the rectangular property.

\footnotetext{
${ }^{8}$ The relationship between the two domain conditions is discussed in the supplementary note that is available online at: http://www.iser.osaka-u.ac.jp/library/dp/2007/DP0699N.pdf

${ }^{9} \mathbb{R}_{+}$and $\mathbb{R}_{-}$denote the set of nonnegative real numbers and nonpositive real numbers, respectively.
} 
Proposition 1. Suppose that $V$ is minimally rich. Then, a social choice function $f$ on $V$ satisfies strategy-proofness and the rectangular property if and only if it is constant.

Proof. Since the "if" part is obvious, we only show the "only if " part. Suppose that $V$ is a minimally rich domain and that a social choice function $f$ satisfies strategyproofness and the rectangular property. In what follows, we will prove the following four claims.

Claim 1. For each $i \in I$, each $v_{-i} \in V_{-i}$, and each $v_{i}, v_{i}^{\prime} \in V_{i}$, if $\sigma_{i}\left(\boldsymbol{v}_{\boldsymbol{i}}, \boldsymbol{v}_{-i}\right)=\boldsymbol{\sigma}_{\boldsymbol{i}}\left(\boldsymbol{v}_{\boldsymbol{i}}^{\prime}, \boldsymbol{v}_{-i}\right)$, then $\boldsymbol{m}_{\boldsymbol{i}}\left(\boldsymbol{v}_{\boldsymbol{i}}, \boldsymbol{v}_{-i}\right)=\boldsymbol{m}_{\boldsymbol{i}}\left(\boldsymbol{v}_{\boldsymbol{i}}^{\prime}, \boldsymbol{v}_{-i}\right)$ : Suppose, by contradiction, that there exist $i \in I, v_{-i} \in V_{-i}$, and $v_{i}, v_{i}^{\prime} \in V_{i}$ such that $\sigma_{i}\left(v_{i}, v_{-i}\right)=$ $\sigma_{i}\left(v_{i}^{\prime}, v_{-i}\right)$ and $m_{i}\left(v_{i}, v_{-i}\right) \neq m_{i}\left(v_{i}^{\prime}, v_{-i}\right)$. Without loss of generality, $m_{i}\left(v_{i}, v_{-i}\right)>$ $m_{i}\left(v_{i}^{\prime}, v_{-i}\right)$. Then agent $i$ with the valuation vector $v_{i}^{\prime}$ can gain by announcing the false valuation $v_{i}$, contradicting strategy-proofness.

Claim 1 states us that given agent $j \neq i$ announce $v_{j}$, agent $i$ 's consumption bundle depends on the type of the indivisible object that she receives. Thus, when agent $i$ is assigned to type- $t$ object, we denote her consumption bundle allocated to agent $i$ by $\left(t, m_{i}^{t}\right)$. Let $O_{i}\left(v_{-i}\right)$ be the option set for agent $i$ given $v_{-i}$, defined by

$$
O_{i}\left(v_{-i}\right) \equiv\left\{t \in T: \exists v_{i} \in V_{i} \text { such that } f_{i}\left(v_{i}, v_{-i}\right)=\left(t, m_{i}^{t}\right)\right\} .
$$

This is the set of the types of indivisible objects that agent $i$ can obtain by changing her valuation vector.

Claim 2. For each $v \in V$, each $i \in I$, and each $t \in T$ such that $f_{i}(v)=\left(t, m_{i}^{t}\right)$, if $\left|O_{i}\left(v_{-i}\right)\right| \geq 2$, then $v_{i}(t)+m_{i}^{t}>v_{i}\left(t^{\prime}\right)+m_{i}^{t^{\prime}}$ for each $\boldsymbol{t}^{\prime} \in \boldsymbol{O}_{\boldsymbol{i}}\left(\boldsymbol{v}_{-i}\right) \backslash\{\boldsymbol{t}\}$ : Suppose, by contradiction, that there exist $v \in V, i \in I$ and $t \in T$ such that $f_{i}(v)=\left(t, m_{i}^{t}\right),\left|O_{i}\left(v_{-i}\right)\right| \geq 2$, and $v_{i}(t)+m_{i}^{t} \leq v_{i}\left(t^{\prime}\right)+m_{i}^{t^{\prime}}$ for some $t^{\prime} \in O_{i}\left(v_{-i}\right) \backslash\{t\}$. Since $t^{\prime} \in O_{i}\left(v_{-i}\right)$, there exists $v_{i}^{\prime} \in V_{i}$ such that $f_{i}\left(v_{i}^{\prime}, v_{-i}\right)=\left(t^{\prime}, m^{t^{\prime}}\right)$. There are two cases.

Case 2-1. $\boldsymbol{v}_{\boldsymbol{i}}(\boldsymbol{t})+\boldsymbol{m}_{\boldsymbol{i}}^{\boldsymbol{t}}<\boldsymbol{v}_{\boldsymbol{i}}\left(\boldsymbol{t}^{\prime}\right)+\boldsymbol{m}_{\boldsymbol{i}}^{\boldsymbol{t}^{\prime}}$ : In this case, we have

$$
v_{i}\left(\sigma_{i}(v)\right)+m_{i}(v)<v_{i}\left(\sigma_{i}\left(v_{i}^{\prime}, v_{-i}\right)\right)+m_{i}\left(v_{i}^{\prime}, v_{-i}\right),
$$

which is a contradiction to strategy-proofness.

Case 2-2. $\boldsymbol{v}_{\boldsymbol{i}}(\boldsymbol{t})+\boldsymbol{m}_{\boldsymbol{i}}^{\boldsymbol{t}}=\boldsymbol{v}_{\boldsymbol{i}}\left(\boldsymbol{t}^{\prime}\right)+\boldsymbol{m}_{\boldsymbol{i}}^{\boldsymbol{t}^{\prime}}$ : Since $t \neq t^{\prime}, f(v) \neq f\left(v_{i}^{\prime}, v_{-i}\right)$. However, since $v_{i}(t)+m_{i}^{t}=v_{i}\left(t^{\prime}\right)+m_{i}^{t^{\prime}}$, we have

$$
\begin{aligned}
v_{i}\left(\sigma_{i}\left(v_{i}^{\prime}, v_{-i}\right)\right)+m_{i}\left(v_{i}^{\prime}, v_{-i}\right) & =v_{i}\left(\sigma_{i}(v)\right)+m_{i}(v), \\
v_{j}\left(\sigma_{j}\left(v_{i}^{\prime}, v_{j}, v_{-i, j}\right)\right)+m_{j}\left(v_{i}^{\prime}, v_{j}, v_{-i, j}\right) & =v_{j}\left(\sigma_{j}\left(v_{i}^{\prime}, v_{j}, v_{-i, j}\right)\right)+m_{j}\left(v_{i}^{\prime}, v_{j}, v_{-i, j}\right) \text { for each } j \neq i,
\end{aligned}
$$

which is a contradiction to the rectangular property.

Claim 3. For each $i \in I$ and each $v_{-i} \in V_{-i},\left|O_{i}\left(v_{-i}\right)\right|=1$ : Suppose, by contradiction, that there exist $i \in I$ and $v_{-i} \in V_{-i}$ such that $\left|O_{i}\left(v_{-i}\right)\right| \geq 2$. Let $t^{\prime} \in O_{i}\left(v_{-i}\right)$. Since $t^{\prime} \in O_{i}\left(v_{-i}\right)$, there exists $v_{i}^{\prime} \in V_{i}$ such that $f_{i}\left(v_{i}^{\prime}, v_{-i}\right)=\left(t^{\prime}, m^{t^{\prime}}\right)$. 
Now, select any of the following:

$$
t^{\prime \prime} \in \arg \max _{t \in O_{i}\left(v_{-i}\right) \backslash\left\{t^{\prime}\right\}} v_{i}^{\prime}(t)+m_{i}^{t} .
$$

Since $t^{\prime \prime} \in O_{i}\left(v_{-i}\right)$, there exists $v_{i}^{\prime \prime} \in V_{i}$ such that $f_{i}\left(v_{i}^{\prime \prime}, v_{-i}\right)=\left(t^{\prime \prime}, m_{i}^{t^{\prime \prime}}\right)$. Let $M \equiv m_{i}^{t^{\prime \prime}}-m_{i}^{t^{\prime}}$. By Claim 2, we have

$$
\begin{aligned}
\Delta v_{i}^{\prime}\left(t^{\prime} ; t^{\prime \prime}\right) & =v_{i}^{\prime}\left(t^{\prime}\right)-v_{i}^{\prime}\left(t^{\prime \prime}\right)>m_{i}^{t^{\prime \prime}}-m_{i}^{t^{\prime}}=M, \\
-\Delta v_{i}^{\prime \prime}\left(t^{\prime} ; t^{\prime \prime}\right) & =v_{i}^{\prime \prime}\left(t^{\prime \prime}\right)-v_{i}^{\prime \prime}\left(t^{\prime}\right)>m_{i}^{t^{\prime}}-m_{i}^{t^{\prime \prime}}=-M .
\end{aligned}
$$

Therefore, $\Delta v_{i}^{\prime}\left(t^{\prime} ; t^{\prime \prime}\right)>M>\Delta v_{i}^{\prime \prime}\left(t^{\prime} ; t^{\prime \prime}\right)$. Since $V$ is minimally rich, there exists $v_{i}^{*} \in V_{i}$ such that (i) $\Delta v_{i}^{*}\left(t^{\prime} ; t^{\prime \prime}\right)=M$ and (ii) $\Delta v_{i}^{\prime}\left(t ; t^{\prime \prime}\right) \geq \Delta v_{i}^{*}\left(t ; t^{\prime \prime}\right)$ for each $t \in T \backslash\left\{t^{\prime}, t^{\prime \prime}\right\}$. Condition (i) implies that

$$
v_{i}^{*}\left(t^{\prime}\right)+m_{i}^{t^{\prime}}=v_{i}^{*}\left(t^{\prime \prime}\right)+m_{i}^{t^{\prime \prime}} .
$$

Condition (ii) and (1) together imply that for each $t \in O_{i}\left(v_{-i}\right) \backslash\left\{t^{\prime}, t^{\prime \prime}\right\}$,

$$
m_{i}^{t^{\prime \prime}}-m_{i}^{t} \geq \Delta v_{i}^{\prime}\left(t ; t^{\prime \prime}\right) \geq \Delta v_{i}^{*}\left(t ; t^{\prime \prime}\right)
$$

Therefore, we have

$$
v_{i}^{*}\left(t^{\prime \prime}\right)+m_{i}^{t^{\prime \prime}} \geq v_{i}^{*}(t)+m_{i}^{t} \text { for each } t \in O_{i}\left(v_{-i}\right) \backslash\left\{t^{\prime}, t^{\prime \prime}\right\} .
$$

By (2) and (3),

$$
v_{i}^{*}\left(t^{\prime}\right)+m_{i}^{t^{\prime}}=v_{i}^{*}\left(t^{\prime \prime}\right)+m_{i}^{t^{\prime \prime}} \geq v_{i}^{*}(t)+m_{i}^{t} \text { for each } t \in O_{i}\left(v_{-i}\right) .
$$

Let $f_{i}\left(v_{i}^{*}, v_{-i}\right)=\left(t^{*}, m_{i}^{t^{*}}\right)$. Thus, strategy-proofness and (4) together imply that

$$
v_{i}^{*}\left(t^{\prime}\right)+m_{i}^{t^{\prime}}=v_{i}^{*}\left(t^{\prime \prime}\right)+m_{i}^{t^{\prime \prime}}=v_{i}^{*}\left(t^{*}\right)+m_{i}^{t^{*}},
$$

which is a contradiction to Claim 2 .

Claim 4. $\boldsymbol{f}(\boldsymbol{v})=\boldsymbol{f}\left(\boldsymbol{v}^{\prime}\right)$ for each $\boldsymbol{v}, \boldsymbol{v}^{\prime} \in \boldsymbol{V}$ : Let $v, v^{\prime} \in V$. By Claim $3, f_{i}\left(v_{i}^{\prime}, v_{-i}^{\prime}\right)=f_{i}\left(v_{i}, v_{-i}^{\prime}\right)$ for each $i \in I$. Therefore, $v_{i}\left(\sigma_{i}\left(v_{i}^{\prime}, v_{-i}^{\prime}\right)\right)+m_{i}\left(v_{i}^{\prime}, v_{-i}^{\prime}\right)=$ $v_{i}\left(\sigma_{i}\left(v_{i}, v_{-i}^{\prime}\right)\right)+m_{i}\left(v_{i}, v_{-i}^{\prime}\right)$ for each $i \in I$. By the rectangular property, we can conclude that $f(v)=f\left(v^{\prime}\right)$.

It can be easily checked the tightness of Proposition $1 .^{10}$ Proposition 1 suggests that strategy-proofness and the rectangular property together imply constancy. However, this impossibility result relies on the domain - on which social choice functions are required to satisfy the two properties - being minimally rich. In fact, unless the domain is minimally rich, there may exist a non-constant social choice function satisfying the two properties. To observe this, we return to Example 1.

\footnotetext{
${ }^{10}$ The independence of axioms is established in the supplementary note that is available online at: http://www.iser.osaka-u.ac.jp/library/dp/2007/DP0699N.pdf
} 
Example 1 (continued). Let $f$ be a social choice function such that for each $v \in V$,

$$
f(v)= \begin{cases}((1,0),(2,0)) & \text { if } v_{1}(1)>1 \\ ((2,1),(1,-1)) & \text { otherwise }\end{cases}
$$

Then, the social choice function $f$ on $V^{*}$ satisfies both strategy-proofness and the rectangular property; however, it is not constant.

Remark 1. Schummer (2000) proposes another domain-richness condition, called monotonic closedness: For each $i \in I$, each $v_{i}^{\prime} \in V_{i}$, and each $t^{\prime} \in T$, there exists $v_{i}^{\prime \prime} \in V_{i}$ such that $\Delta v_{i}^{\prime \prime}\left(t^{\prime} ; t^{\prime \prime}\right)>\Delta v_{i}^{\prime}\left(t^{\prime} ; t^{\prime \prime}\right)$ for each $t^{\prime \prime} \in T \backslash\left\{t^{\prime}\right\}$. It is easy to observe that $V^{*}$ is monotonically closed. Thus, Example 1 implies that a monotonically closed domain is not minimally rich and that on a monotonically closed domain, there exists a non-constant social choice function that is securely implementable.

Based on Saijo, Sjöström, and Yamato (2007) and our proposition, we can obtain that no non-constant social choice function can be securely implementable.

Theorem 1. Suppose that $V$ is minimally rich. Then, a social choice function $f$ on $V$ is securely implementable if and only if it is constant.

\section{Conclusion}

In this study, we applied the notion of secure implementation to the problem of allocating indivisible objects with monetary transfers. We then established that only constant social choice functions on a minimally rich domain can be securely implemented. This negative result suggests that all non-trivial strategy-proof social choice functions do not work well in our environment.

In the following, we discuss a remaining problem. Our study leaves open the question of what class of social choice functions are securely implementable without employing a minimally rich domain. We give a partial answer to the open question. The discussion is based on dual dominance proposed by Saijo (1987). He shows that a social choice function satisfies dual dominance and Maskin monotonicity (Maskin, 1999) if and only if it is constant. Therefore, it is easy to establish that a secure implementable social choice function satisfies dual dominance if and only if it is constant. Furthermore, we can strengthen this result by replacing dual dominance by a weaker condition of social choice functions. ${ }^{11}$ However, it is open that verifying which social choice functions are securely implementable without imposing additional conditions of social choice functions and minimal richness.

\footnotetext{
${ }^{11}$ See for detailed discussion the supplementary note which is available online at: http://www.iser.osaka-u.ac.jp/library/dp/2007/DP0699N.pdf
} 


\section{References}

Alkan, A., G. Demange, and D. Gale (1991) "Fair Allocation of Indivisible Goods and Criteria of Justice," Econometrica 59, 1023-1039.

Bochet, O. and T. Sakai (2007) "Secure Implementation in Allotment Economies," mimeo, Maastricht University and Yokohama National University.

Cason, T., T. Saijo, T. Sjöström, and T. Yamato (2006) "Secure Implementation Experiments: Do Strategy-proof Mechanisms Really Work?" Games and Economic Behavior 57, 206-235.

Fujinaka, Y. and T. Sakai (2006) "Maskin Monotonicity in Economies with Indivisible Goods and Money," Economics Letters 94, 253-258.

Maskin, E. (1999) "Nash Equilibrium and Welfare Optimality," Review of Economic Studies 66, 23-38.

Mizukami, H., and T. Wakayama (2007) "Full-truthful Implementation in Nash Equilibria," Research Paper Series No.27, Tokyo Metropolitan University.

Saijo, T. (1987) "On Constant Maskin Monotonic Social Choice Functions," Journal of Economic Theory 42, 382-386.

Saijo, T., T. Sjöström, and T. Yamato (2007) "Secure Implementation," Theoretical Economics 2, 203-229.

Schummer, J. (2000) "Manipulation through Bribes," Journal of Economic Theory 91, 180-198.

Svensson, L.G. (1983) "Large Indivisibles: An Analysis with Respect to Price Equilibrium and Fairness," Econometrica 51, 939-954. 